exams as well as practising dentists.

As a practising clinician I thoroughly enjoyed reading this book and learnt a lot of tips that are useful in daily practice. I was impressed by the breadth of topics covered under dental materials in this book.

This may not be intended to serve as a definitive dental materials or restorative text, but very successfully fulfils its aim by bridging the gap between dental material science and clinical practice.

R. Paul

\section{ORAL AND MAXILLOFACIAL} TRAUMA, 4TH EDITION

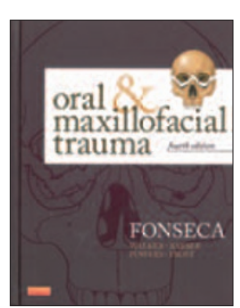

R. J. Fonseca, R. V. Walker, H. D. Baxter, M. P. Powers, D. E. Frost

Elsevier

price $€ 230.00$; pp 912

ISBN 9781455705542

This fourth edition American text has evolved from two-volumes to a convenient, single volume, transforming it into a more accessible form. Despite this reduction, it does not fail to comprehensively include all the salient topics relevant to the management of oral and maxillofacial trauma. Currently this 2013 text is the most up to date reference available on this topic with the classical British equivalent by Rowe and Williams being almost 20 years old and out of print. For those who prefer their text in megabytes rather than pages, there is the option to purchase the text in a digital format.

The 913 page, A4 hardback is crammed with tried-and-tested traditional concepts such as open reduction and fixation techniques for fractures as well as references to modern techniques including surgical navigation, intra-operative radiography and endoscopic repair. Despite being compiled by over 80 individual authors, the narrative is clear and consistent throughout. The photographs and diagrams are of a high quality and with the tables provide a concise overview of the topic. Each chapter has extensive references that are useful for anyone preparing for exams.

Despite its seemingly specific title, the first 20\% covers general but relevant aspects of trauma management includ- ing the physiological impact of trauma and the current important features of the advanced trauma life support (ATLS) approach to emergency care. The following $60 \%$ is then devoted to the management of head and neck injuries; over half of this material expectedly describes the management of the commonly encountered bone and soft tissue injuries. Included within this is the specific management of bites, salivary gland trauma and ophthalmic consideration. The complex specialty of ophthalmology has been tailored appropriately to be relevant to oral and maxillofacial trauma whilst managing to retain sufficient depth and detail. The final 20\% of the text covers interesting special considerations including ballistics and missile injuries, burns and endoscopic approaches to trauma surgery.

The scope and depth of this text makes it ideal for trainee and consultant surgeons alike. However, it is a useful tome for anyone who is interested in advancing their knowledge of oral and maxillofacial traumatology including general dental practitioners who are often the first to triage milder trauma cases. An extensive chapter on dento-alveolar trauma based on the esteemed work of Jens Andreason ensures the text has relevance to the entire dental team.

P. Saini

\section{SURGICAL ESSENTIALS OF IMMEDIATE IMPLANT DENTISTRY}

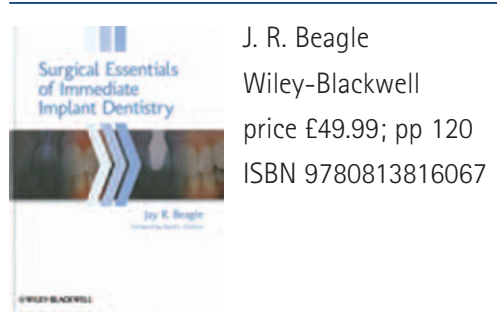

The intention of this text is to provide clinicians with the fundamental evidence-based information necessary to incorporate this important modality into their patient care. This is achieved admirably by the author.

This modern hardcover book is a clear, concise and comprehensive read. It is comprised of eight chapters, the majority of which being subdivided into subheadings. Each chapter is well written giving the reader appropriate relevant information with a well-structured and easy-to- follow layout. The content is supported by up-to-date accurate references and where appropriate, it highlights areas for future research. It contains more than 250 high-quality clinical photographs, radiographs and tables.

The introduction discusses the historical development of the immediate implant placement into an extraction socket, the predictable success of this procedure and the importance of evidenced-based practice. Chapter two provides a detailed description of the pre-operative risk assessment. It helps the reader to comprehend all the essential parameters that need to be evaluated prior to immediate implant placement to preclude any pitfalls. In particular, dentists' experience, patients' local and systemic factors, biomechanics of the chosen implant system and grafting materials. The SAC classification for surgical and prosthetic applications for implant dentistry is used for risk assessment. In chapter three and four the author discusses the indications and contraindications of immediate surgical implant placement, making suggestions on how to assess potential implant site with more accuracy. The fifth chapter covers the healing of extraction site with reference to animal and human studies. Chapter six focuses on the various surgical techniques and the appropriate use of instruments to minimise trauma to the extraction socket wall preserving the hard and soft tissues. Chapter seven is focused on salient aspects of immediate placement technique and finally, the last chapter concentrates on complications of the surgical procedure and implant failure.

of note, the dental notation system used in this book is the universal numbering system commonly used in the United States, as it is the origin of this book.

In summary, this is a well-written, comprehensive, clinically relevant and evidence-based book of surgical implant placement into the sockets of immediately removed teeth. This book is a suitable read for a wide variety of readers including postgraduate students, dental professionals with particular interest in implant dentistry, specialists and subspecialty consultants.

E. Kyriakidou 